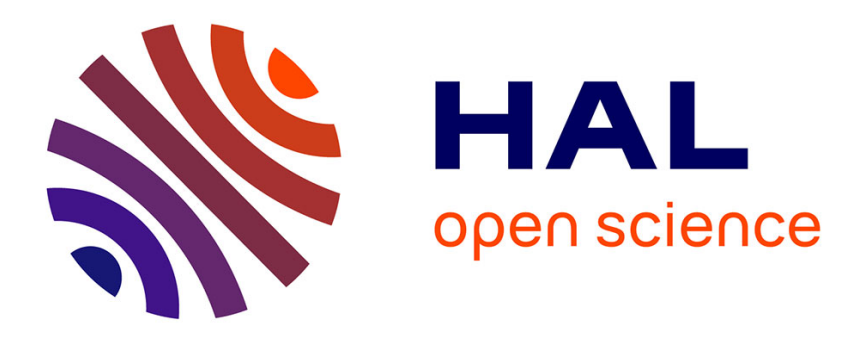

\title{
Spectral Approach to Displacement Evaluation From Image Analysis
}

Babacar Wagne, Stéphane Roux, François Hild

\section{To cite this version:}

Babacar Wagne, Stéphane Roux, François Hild. Spectral Approach to Displacement Evaluation From Image Analysis. European Physical Journal: Applied Physics, 2002, 17, pp.247-252. 10.1051/epjap:2002019 . hal-00002903

\section{HAL Id: hal-00002903 https://hal.science/hal-00002903}

Submitted on 20 Sep 2004

HAL is a multi-disciplinary open access archive for the deposit and dissemination of scientific research documents, whether they are published or not. The documents may come from teaching and research institutions in France or abroad, or from public or private research centers.
L'archive ouverte pluridisciplinaire HAL, est destinée au dépôt et à la diffusion de documents scientifiques de niveau recherche, publiés ou non, émanant des établissements d'enseignement et de recherche français ou étrangers, des laboratoires publics ou privés. 


\title{
Spectral Approach to Displacement Evaluation From Image Analysis
}

\author{
Babacar Wagne, ${ }^{1}$ Stéphane Roux ${ }^{1}$ and François Hild ${ }^{2}$ \\ 1 Laboratoire "Surface du Verre et Interfaces", UMR CNRS/Saint-Gobain, \\ 39 Quai L. Lefranc, F-93303 Aubervilliers Cedex, France. \\ 2 LMT-Cachan, ENS de Cachan/UMR CNRS/Université Paris 6 \\ 61 Avenue du Président Wilson, F-94235 Cachan Cedex, France.
}

Received: date / Revised version: date

\begin{abstract}
A new approach to determine the displacement field between a reference and a deformed image is introduced. The method is based on a spectral decomposition of the displacement field which is determined through a multi-scale approach. The method is tested here on an artificial example by using computergenerated images devoid of boundary effects (i.e., periodic boundary conditions are used in the image texture and displacement field). The performance of the proposed algorithm allows for the determination of the prescribed displacement field up to machine precision, for a strain field whose trace extends from $-50 \%$ to $40 \%$ with a root-mean-square average of $20 \%$, within sub-minute runs on a standard PC.
\end{abstract}

PACS. $46.80 .+\mathrm{j}$ Measurement methods and techniques in continuum mechanics of solids -06.30. Bp Spatial dimensions (e.g., position, lengths, volume, angles, and displacements) - 07.05.Pj Image processing 42.30.Va Image forming and processing - 46.15.-x Computational methods in continuum mechanics

\section{Introduction}

In many applications, computer vision is used to detect and follow the motion of objects (e.g., robotics, medical imaging, meteorology, and experimental visualization in fluid or solid mechanics). The technological development of digital image recorders (e.g., CCD camera or video recorder), both in quality and low-cost motivates the design of versatile, flexible, robust and precise means of measuring displacement or velocity fields.

The measurement of the apparent $2 \mathrm{D}$ motion in an image sequence is performed by pattern matching of various 'markers' in consecutive images. A black and white image sequence is represented by a gray level function or intensity. Under a constant illumination, the intensity is thus passively advected by a velocity field to be determined, but remains otherwise unchanged. This 'conservation' principle allows one to write an equation obeyed by the image intensity in time, namely the so-called 'optical flow' equation. Used in differential form, this conservation principle has given a wide body of efficient numerical algorithms used to extract the velocity field. Alternatively, the same conservation principle can be used through a direct matching of sub-regions within the original images.

Let us note that the measurement of the displacement is an ill posed problem. The displacement can only be measured along the direction of the intensity gradient. Consequently, additional hypotheses have to be proposed to solve the problem. If one assumes a locally constant displacement (or velocity), a block matching procedure is found. It consists in maximizing the cross-correlation function $[1,2]$. This method is, for instance, used to measure heterogeneous displacement fields in solid mechanics $[3,4]$. Many of the procedures available nowadays are based on Correlation Image Velocimetry (CIV) methods [5] directly inspired from earlier developments in fluid mechanics [6, 7]. Conversely, variational formulations can be used. They are mainly based on the optical flow equation. A spatial regularization was introduced by Horn and Schunck [8] and consists in a looking for smooth displacement solutions. However, this method is not appropriate for problems dealing with discontinuities in the apparent displacement [9]. In the latter case, the quadratic penalization is replaced by 'smoother' penalizations based on robust statistics [10-12]. Furthermore, when dealing with deformable solids, other regularization techniques are to be introduced such as the one based on the strain energy [13]. It can be noted that problems as complex as face tracking with three dimensional motions and deformations can be handled by using adapted procedures [14].

Similarly, some attempts have been proposed to go beyond the simple cross-correlation of sub-images extracted from the reference and deformed images [15,16]. Based on finite-element determination of the displacement field, they suffer from the generic existence of many minima 
of the objective function, so that the determination of a global optimum is a cost-effective and sometimes insecure operation. Even up-to-date algorithms suited to such multi-minima optimization problems, such as simulated annealing or genetic optimization cannot guarantee both a reliable and cost-effective solution [17].

In this paper, a novel approach, whose capabilities appear as promising, is proposed. This is a preliminary report on the feasibility of the method, which has not yet been validated over a wide range of cases. In particular, common problems such as the loss of accuracy at the edge of the image are avoided by testing only periodic images (paving the plane without discontinuities) which are deformed through periodic displacement fields. This special class of problem is easily tractable with the proposed method, which is based on an intensive use of Fourier transforms. A concrete example will demonstrate that even difficult cases can be treated by the present approach with a good accuracy. However, some more effort has to be devoted to the treatment of edge effects and to the development of a robust procedure to get a good solution in most practical cases.

\section{Problem}

The spirit of the method is similar to the one-dimensional treatment proposed by Roux et al. [18], but developed in two dimensions. As mentioned earlier, the discussion is restricted to periodic boundary conditions, suited for the spectral approach presented herein. However, in Ref. [18], extensions of the present approach have been presented to consider non-periodic test functions (e.g., affine functions), which allowed for a systematic treatment of more general strain fields in one dimension. Similar extensions are still to be developed for the two dimensional case, yet the road map is clear since the general framework developed herein is valid for any trial displacement, not only that corresponding to a spectral decomposition of the displacement field. The present paper gives the general outline of the method, and for the sake of simplicity, in this first study, the boundary effects are deliberately ignored, and thus, only a periodic case is considered.

First, let us consider a reference image, defined as $f(\underline{x})$, e.g., a gray level distribution. This function is assumed to be periodic of period $L$ both in $x$ and $y$ directions, where $L$ is the 'size' of the image expressed in pixels. An inplane displacement field $\underline{U}(\underline{x})$ is defined. Similarly, this displacement field is assumed to be periodic in both directions with the same period. The passive advection of the texture $f$ by the displacement field defines a 'deformed image', $g(\underline{x})$, such that

$$
g(\underline{x}+\underline{U}(\underline{x}))=f(\underline{x}) .
$$

This equation is the integral form of the 'optical flow equation.' Note that this choice prevents the occurrence of large scale overall rotations, although, locally, rotations are allowed.
The problem to address is the determination of the displacement field $\underline{U}$ from the exclusive knowledge of $f$ and $g$. As such, the problem is ill posed, unless additional assumptions are made on the regularity of the displacement field so that the information is sufficient to determine $\underline{U}$ with a reasonable accuracy [8]. As a particular case, one can note that if $\underline{U}$ is simply a rigid body translation, then the problem can be addressed by using the standard crosscorrelation technique to maximize the overlap between the translated $f$ and $g[2,18]$. Alternatively, if no information on the regularity of $\underline{U}$ is available, then there are more unknowns than equations, so that even ignoring the problem of multiplicity of local minima, the problem cannot be solved. Here, the basic assumption is that the displacement field has dominant long-wavelength components, and for practical purposes, it is assumed to be low-pass filtered. The translation case thus appears to be the extreme limit of preserving only one mode (i.e., only the wavenumber $k=0$ has a non-zero amplitude).

\section{2D Spectral Method}

Let us introduce the following objective functional, $\mathcal{T}$, operating on displacement fields $\underline{V}(\underline{x})$

$$
\mathcal{T}[\underline{V}]=\iint(g(\underline{x}+\underline{V}(\underline{x}))-f(\underline{x}))^{2} \mathrm{~d} \underline{x} .
$$

This functional reaches its minimum value, 0 , for $\underline{V}=\underline{U}$ (see Eq. (1)), up to a rigid translation of an arbitrary integer number of periods in both directions. This functional is the generalization of that used by Roux et al. [18] to study one-dimensional signals.

The trial displacement $\underline{V}$ is assumed to be $L$-periodic in both directions, since this is a basic prerequisite. Thus, it will appear as convenient to work in Fourier space, defining the Fourier transform $\widetilde{h}$ of any function $h$ as

$$
\widetilde{h}(\underline{k})=\iint h(\underline{x}) \exp (i \underline{k} \cdot \underline{x}) \mathrm{d} \underline{x} .
$$

To fulfill the smoothness assumption on $\underline{U}, \underline{V}$ is low-pass filtered. The space of periodic vector displacement fields $E_{K}$ is introduced such that $\widetilde{V}(\underline{k})=0$ if $|\underline{k}|<K$. Other choices have been proposed in the literature in order to match specific requirements. Wavelet functions as proposed in particular by Bernard [19] are well suited to such a problem.

Let us first assume that $f$ and $g$ are sufficiently smooth on small scales, and the displacement small enough in amplitude so that a Taylor expansion of $g$ up to the first order can be introduced in the above expression for $\mathcal{T}$

$$
\mathcal{T}\left[\underline{V]}=\iint(g(\underline{x})-f(\underline{x})+\underline{V}(\underline{x}) \cdot \underline{\nabla} g(\underline{x}))^{2} \mathrm{~d} \underline{x} .\right.
$$

It can be noted that there is no need to add a penalization condition to ensure the smoothness of the solution [8] since the trial displacements fulfill this requirement. Nevertheless, it is important to note that the Taylor expansion requires explicitly that $g$ itself is sufficiently smooth. 
This may appear as a rather stringent requirement since one may not always master the small scale behavior of the texture of the reference image. We will see in the following that the proposed procedure allows us to deal with texture which finally are not extremely smooth (even nondifferentiable) provided they have a sufficiently rich power spectrum. The latter property will allow us to restore part of the required smoothness after filtering while still preserving the needed information. Equation (4) corresponds to the objective functional associated to the optical flow equation. The displacement field is decomposed as a linear combination of a basis of $E_{K}, \underline{V}(\underline{x})=\sum_{j} \underline{v}_{j} \Psi_{j}(\underline{x})$, so that $\mathcal{T}[\underline{V}]$ becomes a quadratic form in the (vector) amplitudes $\underline{v}_{j}$. The extremality condition thus dictates, for all $j$,

$$
\begin{gathered}
\left(\iint(\underline{\nabla g} \otimes \underline{\nabla g})(\underline{x}) \Psi_{j}(\underline{x}) \Psi_{k}(\underline{x}) \mathrm{d} \underline{x}\right) \underline{v}_{k} \\
=\iint(f(\underline{x})-g(\underline{x})) \underline{\nabla} g(\underline{x}) \Psi_{j}(\underline{x}) \mathrm{d} \underline{x} .
\end{gathered}
$$

This system will be written in a matrix form for simplicity in the sequel, as

$$
\underline{\underline{M}}_{j k} \underline{v}_{k}=\underline{A}_{j} .
$$

One can note that the dyadic product $(\underline{\nabla g} \otimes \underline{\nabla g})$ by itself cannot be inverted (it has always a zero eigenvalue in the direction normal to the gradient of $g$ ), and hence this formula cannot be used to determine $\underline{V}$, if $\Psi$ tends to a Dirac distribution, as anticipated from the remark on the necessary regularity of $\underline{V}$. However, if $E_{K}$ is chosen to be restricted to wavelengths much greater than the correlation length of the texture, the left hand side operator becomes a genuine definite positive operator, and the product with $\Psi \Psi$ is nothing but a filtering of the operator $(\underline{\nabla g} \otimes \underline{\nabla g})$.

In the following, a spectral representation for the displacement field is used. In the present case, the function $\Psi_{k}(\underline{x})$ is equal to $\exp (i \underline{k} . \underline{x})$ (this complex notation implies that the objective functional is the integral of the squared modulus, and hence conjugation will appear in Eq. (5)). The matrix element $M_{k k^{\prime}}$ can be written as

$$
M_{k k^{\prime}}=(\underline{\nabla g} \widetilde{\nabla} \underline{\nabla} g)\left(k-k^{\prime}\right)
$$

and thus $\underline{M} \cdot \underline{v}$ is a convolution product in Fourier space. However, it appears as being awkward to perform such a convolution. If one reverts to real space, then the convolution appears to be a simple product (i.e., a diagonal operator). However, the use of a Fourier transform is a useful step for the filtering operation. Similarly, the second member is the filtered (i.e., restriction to $E_{K}$ space) of the direct product $(f-g) \underline{\nabla g}$. Therefore, the following scheme can be proposed:

1. Compute the tensor field $\underline{\nabla g} \otimes \underline{\nabla g}$, Fourier transform it and preserve only the modes $|\underline{k}|<K$. Inverse Fourier transform the latter, to obtain a real space tensor field, $\underline{M}(\underline{x})$. The latter needs only to be computed over a $\bar{c}$ coarse mesh of size $2 K \times 2 K$ instead of the original $L \times L$ one.
2. Compute the vector field $(f-g) \underline{\nabla} g$, Fourier transform it and preserve only the modes $|\underline{k}|<K$. Inverse Fourier transform the latter, to obtain a real space vector field, $\underline{A}(\underline{x})$, on the coarse mesh.

3. Compute the vector field $\underline{B}(\underline{x}) \equiv \underline{M}^{-1}(\underline{x}) \underline{A}(\underline{x})$, after checking that the tensor $\underline{M}$ can indeed be inverted.

4. Fourier transform the $\underline{B}(\underline{\bar{x}})$ field using the relevant $K$ modes. The latter components give directly the unknown $\underline{v}_{k}$ components

$$
\underline{v}_{k}=\underline{\widetilde{B}}(\underline{k})
$$

\section{Discussion}

The above procedure is extremely simple, both in its principle and in its implementation. It is also very efficient in terms of computer time since it relies heavily on FFT codes, and requires only a $2 \times 2$ tensor inversion on a coarse mesh. However, the very basic hypothesis used to derive such a procedure has not yet been discussed, i.e., the validity of the Taylor expansion used in Eq. (4). This question is a rather subtle issue. Generically, the method is to be used on a texture $f$ or $g$ which is supposed to have short range correlations, and hence, if the displacement amplitude is greater than this correlation length, the Taylor expansion is inadequate. Moreover, the $\underline{\underline{M}}$ tensor is only invertible when $\nabla g$ is rapidly varying over scales of the order of $L / K$. To deal with this problem, the following approach can be used:

A. Use a texture such that a long-range correlation exists. This will allow one to low-pass filter the original and deformed images. This first filtering is performed so that the rapid fluctuations of $f$ and $g$ are erased over a scale such that the amplitude of the anticipated displacement is less than the shorter wavelength preserved in this filtering. That is, an upper wavenumber, $K^{\prime}$, is used such that $U<L / K^{\prime}$ where $U$ is the magnitude of the anticipated displacement. This guarantees that the Taylor expansion is legitimate. The determination of the displacement field is then carried out with $K$ modes, by using the condition $K \ll K^{\prime}$. The criterion to be used to adjust $K$ is that the minimum eigenvalue of $M$ is greater than a minimum threshold.

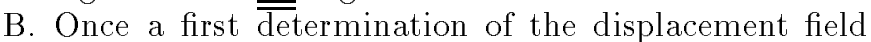
has been obtained with a good accuracy, the reference (or deformed) image is corrected by using this displacement field. Two images are obtained for which the relative displacement field is much smaller in amplitude. This allows for a relaxation of the constraint on $K^{\prime}$ (i.e., use a larger value) and thus to expand the number of modes $K$ used to determine $\underline{V}$.

C. This sequence of operations is repeated as many times as required to estimate the precision of the solution using the objective functional $\mathcal{T}$. 

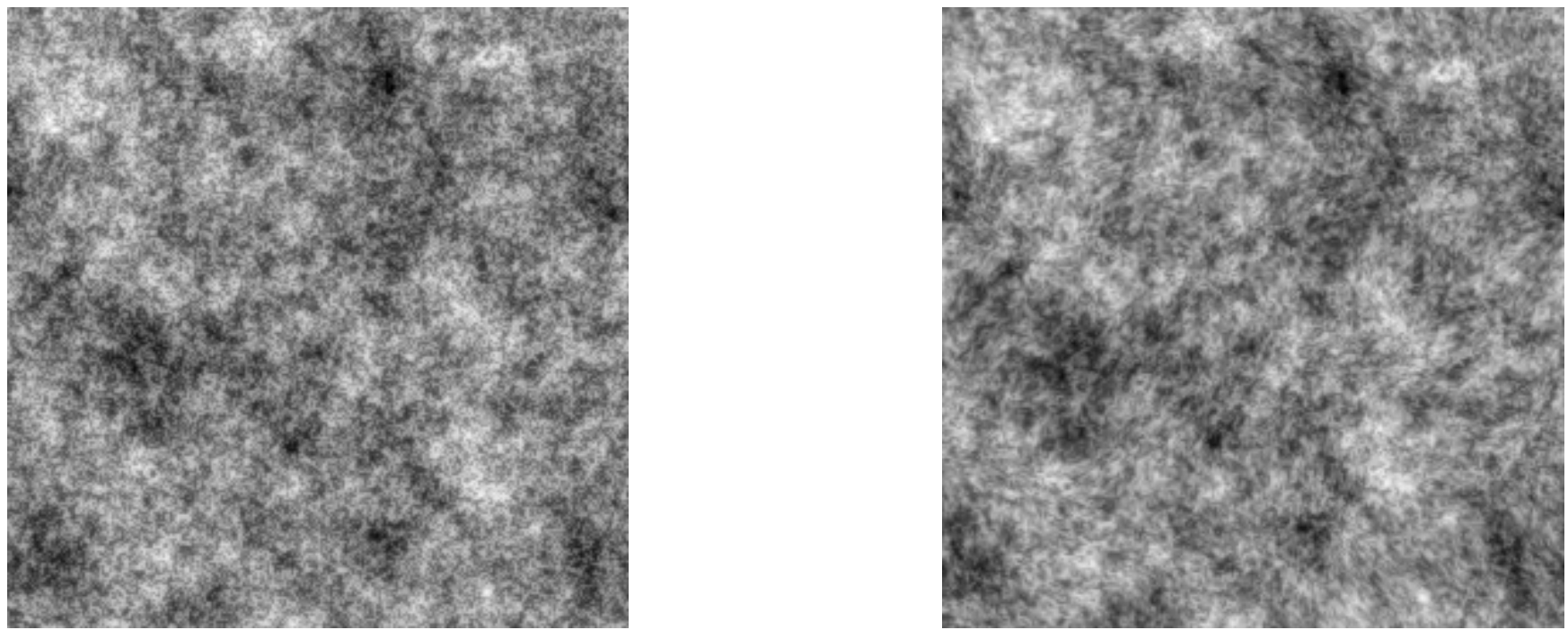

Fig. 1. Reference (left) and deformed (right) images which allow for the determination of the displacement field from their comparison. The images are $256 \times 256$ gray-coded with periodic boundary conditions. The gray level is encoded as a single precision real number.

\section{A practical example}

To validate the method, an artificial test case is generated numerically. First a random texture, $f(\underline{x})$, is created. A self-affine texture is chosen with a roughness exponent $\zeta$ taken to be 0 , so that the amplitude of the Fourier transform scales with the wavenumber as $|\tilde{f}(\underline{k})|^{2} \propto|\underline{k}|^{-2}[20]$. The image size $L$ was taken to be 256 pixels. An example of such a texture is shown in Fig. (1-left). This choice is motivated by remark A. of the previous section.

The displacement field was also chosen to be have dominant components for low wavenumbers. The chosen displacement field has a number of Fourier modes $K=10$, i.e., $\tilde{U}(\underline{k})=0$ for $|\underline{k}|>K$. An example is shown in Fig. (2). For low wavenumbers, the power-spectrum of $\underline{U}$ was chosen to be also self-affine with a roughness exponent, $\zeta_{u}=1$, thus the power spectrum of $\underline{U}$ decays on average as $|k|^{-4}[20]$. In other words, the strain field is a white noise field.

Once the displacement field and initial texture have been generated, the deformed image is computed by using Eq. (1), and a linear interpolation of the gray levels to evaluate the pixel value in the deformed image. The obtained $g$ image is shown in Figure (1-right).

The displacement field shown in Figure (2) is drawn with no amplification. The RMS displacement per site is 6.2 pixels, and the maximum displacement amplitude is 13 pixels. However, the local strain is quite large. Figure (3) shows the map of the volumetric and deviatoric components of the strain, defined as

$$
\begin{aligned}
& \varepsilon_{\text {vol }}=\operatorname{tr}(\underline{\underline{\varepsilon}})=\underline{\nabla} \cdot \underline{U} \text { and } \\
& \varepsilon_{\text {dev }}=\sqrt{\operatorname{tr}\left(\underline{\underline{\varepsilon}}-(1 / 2) \operatorname{tr}(\underline{\underline{\varepsilon}}) \underline{\underline{1}}^{2}\right.}
\end{aligned}
$$

where $\underline{\underline{\varepsilon}}$ is the infinitesimal strain tensor, $\varepsilon_{i j}=(1 / 2)\left(\partial_{i} U_{j}+\right.$ $\left.\partial_{j} U_{i}\right)$. Even though strain levels are greater than a few

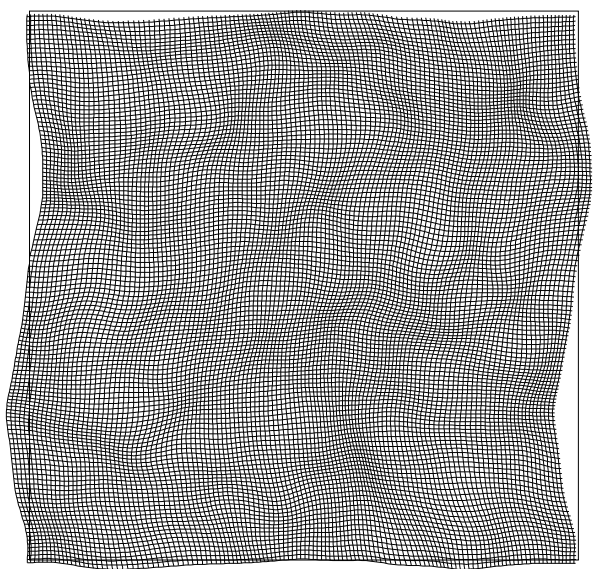

Fig. 2. Displacement field prescribed on a regular square mesh. Note that the displacement is reproduced without any amplification factor (unit scale). The computed displacement field is not shown because one cannot distinguish it from the prescribed one. The RMS difference in the displacement field is of the order of $10^{-6}$ pixel, i.e., it reaches the machine precision (i.e., single precision in a Fortran code).

percents, the infinitesimal strain tensor can be used (it then corresponds to the nominal strain tensor when no body rotations occur).

The volumetric strain ranges from $-52 \%$ to $42 \%$, with an RMS value of $15 \%$, and the deviatoric strain ranges from 0 to $43 \%$. It is to be emphasized that such amplitudes of strains are far beyond the possibility of most classical digital image correlation (DIC) approaches [21]. In practice, such tools are used for strains that rarely exceed a few percents. Although it is an ideal case, where no noise is present at any stage of the computation, since all generation and analysis are performed numerically, the chosen 

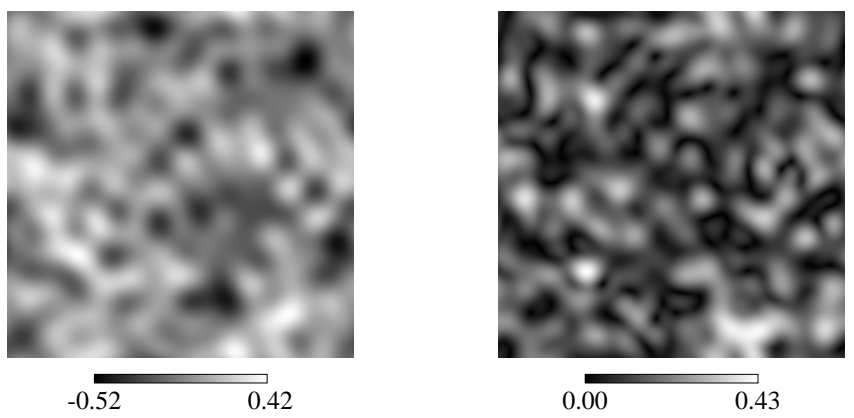

Fig. 3. Volumetric (left) and deviatoric (right) strain fields that show the difficulty of this test case. Note the gray level scale that is indicative of the amplitude of strain values. The strain maps are computed from the prescribed displacement field shown in Figure (2).

test case is very difficult and it would not have been easily resolved by using standard DIC approaches.

Starting from the two images $f$ and $g$, shown in Fig. (1), the determination of the displacement field is carried out recursively as explained in the previous sections. To start off with, a very low number of modes, $K=2$, is considered and every 18 th iteration, 2 additional modes are added, so that for the 18 th iteration, $K=4$, and finally for the 72 nd iteration, $K=10$ modes are allowed, as used in the original generation of the displacement field.

Let us note that as a variation with the general procedure outlined above, no filtering of the texture was used, because it was chosen to contain initially sufficient longrange correlations to render this additional smoothing operation unnecessary. The fact that filtering is not needed constitutes an additional hint that the method is quite robust. It also suggests that some more work should be devoted to clarifying the required properties of the texture to have a viable procedure with a large class of displacement fields.

Figure (4) shows the change of the error, (estimated by the objective functional $\mathcal{T}$ ) in $\log$ scale, with the iteration number. A progressive reduction can be observed, which displays a faster convergence when $K$ is increased (every 18th iteration), up to the point where the value of $K$ used in the determination of $\underline{V}$, reaches the value used to generate the displacement field (i.e., $K=10$ ). From this point on, the error suddenly drops very quickly to reach the machine's precision (single precision real numbers used in the program). Consequently, the determined displacement map matches exactly the prescribed one. This result demonstrates the feasibility of the proposed algorithm, in a test case which was a priori difficult to resolve, by using the standard tools.

The program used to perform this analysis was written in Fortran, and the runs were performed on a PC (Pentium III). The computation time needed to perform 100 iterations was less than one minute. Moreover, the present work is devoted to demonstrating the feasibility of the proposed method, and no effort was made to optimize either time or memory. There are many ways to make this procedure faster, in particular, by exploiting the coarse-graining

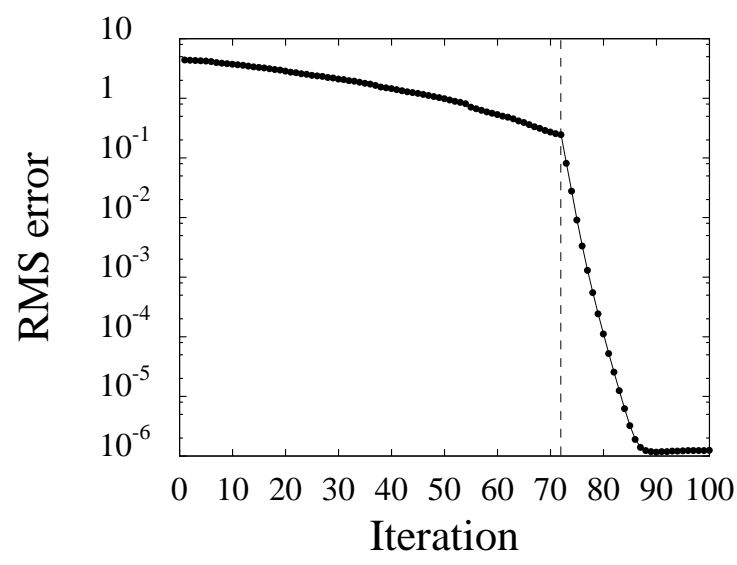

Fig. 4. Norm (RMS per pixel) of the difference between prescribed and computed displacement field as a function of iteration number. More and more modes are included as the computation proceeds, and the sudden final drop corresponds to the stage where the number of allowed modes in the determination equals the number of modes set in the prescribed field.

capabilities of the different steps. Such an approach has been followed by using a more classical cross-correlation approach, and it yielded a gain both in computer time, and in robustness of the method to analyze tests on glass wool samples [22].

\section{Summary}

A novel algorithm has been introduced for the determination of displacement fields relating a reference and deformed image. Only cases with periodic texture and displacement field are considered to work simply with Fourier transforms, although the method can be extended to other cases, since the general framework developed herein is applicable to any class of trial displacement fields.

A test case was studied with large strains reaching $50 \%$ on a $256 \times 256$ pixel image, and yet the displacement field was determined with single precision accuracy within less than 100 iterations, requiring less than a minute of computation on a $\mathrm{PC}$.

A number of extensions have still to be developed, such as an optimized procedure to progressively allow for a larger number of Fourier modes, optimization of the texture to achieve good convergence with the widest class of displacement fields, proper account of edge effects. The success of the present test case indicates that further developments can be carried out along the lines developed herein.

The authors acknowledge useful discussions with Professor Y. Berthaud. 


\section{References}

1. P.J. Burt, C. Yen and X. Xu. Local correlation measures for motion analysis: a Comparative study, in Proc. of the Conf. on Pattern Recognition and Image Processing, Las Vegas, 1982, 269

2. M. A. Sutton, W. J. Wolters, W. H. Peters, W. F. Ranson and S. R. McNeill, Determination of Displacements Using an Improved Digital Correlation Method, Im. Vis. Comp. 1 [3] (1983) 133.

3. A. Lagarde, Advanced Optical Methods and Applications in Solid Mechanics, in: Solid Mechanics and its Applications, (Kluwer, Dordrecht (the Netherlands), 2000), 82.

4. P. K. Rastogi, Photomechanics, in: Topics in Applied Physics, (Springer, Berlin (Germany), 2000), 77.

5. W. Merzkirch, Flow Visualization, (Academic, New York (USA), 1987).

6. T. D. Dudderar and P. G. Simpkins, Laser Speckle Photography in a Fluid Medium, Nature 270 (1977) 45.

7. C. J. D. Pickering and N. A. Halliwell, Speckle Laser in Fluid Flows: Signal Recovery with Two-Step Processing, Appl. Opt. 23 (1984) 1129.

8. B.K.P. Horn and B.G. Schunck, Determining Optical Flow, Artificial Intelligence 17 (1981) 185.

9. A. Mitiche and P. Bouthemy, Computation and Analysis of Image Motion: a Synopsis of Current Problems and Methods, Int. J. Comp. Vision. 19 [1] (1996) 29.

10. P.J. Hubert, Robust Statistics. (Wiley, New York (USA) 1981).

11. M. Black, Robust Incremental Optical Flow, Ph.D. dissertation, Yale University, 1992.

12. J.-M. Odobez and P. Bouthemy, Robust Multiresolution Estimation of Parametric Motion Models, J. Visual Comm. Image Repres. 6 [4] (1995) 348.

13. D. Bogen and D. Rahdert, A Strain Energy Approach to Regularization in Displacement Field Fits of Elastically Deforming Bodies, IEEE Trans. Pattern Analysis and Machine Intelligence, 18 [6] (1996) 629.

14. D. DeCarlo and D. Metaxas, Optical Flow Constraints on Deformable Models with Application to Face Tracking, Int. J. Comp. Vision. 38 [2] (2000) 99.

15. S. Mguil, F. Morestin and M. Brunet, Mesure des déformations par corrélation directe d'images numériques, in: $\mathrm{Y}$. Berthaud, M. Cottron, F. Morestin, P. Moucheront and M. Taroni, eds., Proceedings Photomécanique 98, (GAMAC, 1998), 361.

16. M. A. Sutton, S. R. McNeill, J. D. Helm and Y. J. Chao, Advances in Two-Dimensional and Three-Dimensional Computer Vision, in: Photomechanics, P. K. Rastogi, ed., (Springer, Berlin (Germany), 2000), 323.

17. P. Tokumaru and P. Dimotakis, Image Correlation Velocimetry, Exp. Fluids 19 (1995) 1.

18. S. Roux, F. Hild and Y. Berthaud, Correlation Image Velocimetry: A Spectral Approach, Appl. Optics (2001) in press.

19. C. Bernard, Ondelettes et problèmes mal posés : la mesure du flot optique et l'interpolation irrégulière, Ph.D. dissertation, Ecole Polytechnique, 2001.

20. J. Feder, Fractals (Plenum Press, New York (USA) 1988).

21. L. Chevalier, S. Calloch, F. Hild and Y. Marco, Digital Image Correlation used to Analyze the Multiaxial Behavior of Rubber-Like Materials, Eur. J. Mech. A/Solids 20 (2001) 169.
22. F. Hild, B. Raka, M. Baudequin, S. Roux and F. Cantelaube, Multi-Scale Displacement Field Measurements of Compressed Mineral Wool Samples by Digital Image Correlation, submitted for publication (2001). 